\title{
铜乙二胺法测定中国手工纸纤维素特性粘度的改进实验
}

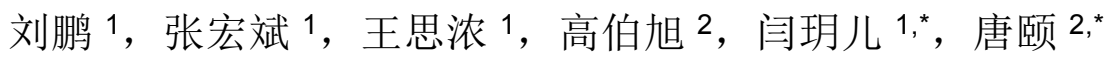 \\ 1 复旦大学图书馆中华古籍保护研究院, 上海 200433 \\ 2 复旦大学化学系, 上海 200433
}

摘要: 介绍了一个通过铜乙二胺法测定纤维素特性粘度的改进实验。在该方法中, 木质素对纤维素特性粘度的测定
会产生巨大影响。为了探讨这一问题, 本实验构建纤维素-木质素模型, 讨论了质素含量对纤维素特性粘度的影
响, 并采用改进的计算方法获得了更加准确的纤维素特性粘度和聚合度。此外, 还探讨了与空气接触时, 不同溶解
时间对纤维素铜乙二胺特性粘度的影响。该实验是纤维素相关材料的基本表征实验, 学生可以了解纤维素的基本性
质和溶解机理, 以及高分子材料粘度的测定和粘度计的使用方法, 还可以学习构建简单模型对各组分影响进行探讨。

关键词: 纤维素; 木质素; 铜乙二胺; 特性粘度

中图分类号: G64; 06

\section{Improvement on Measuring the Intrinsic Viscosity of Cellulose by the Copper Ethylenediamine Method}

\author{
Peng Liu ${ }^{1}$, Hongbin Zhang ${ }^{1}$, Sinong Wang ${ }^{1}$, Boxu Gao ${ }^{2}$, Yueer Yan 1, ${ }^{*}$, Yi Tang ${ }^{2, *}$ \\ ${ }^{1}$ Institute for Preservation and Conservation of Chinese Ancient Books, Fudan University Library, Fudan University, \\ Shanghai 200433, China. \\ ${ }^{2}$ Department of Chemistry, Fudan University, Shanghai 200433, China.
}

\begin{abstract}
An improved experiment for determining the intrinsic viscosity of cellulose through the copper ethylenediamine method is introduced. In this method, lignin has a great impact on determination of the intrinsic viscosity of cellulose. In order to explore this issue, a cellulose-lignin model was constructed to discuss the influence of lignin content on intrinsic viscosity of cellulose. Moreover, an improved calculation method was used for cellulose in this model to obtain a more accurate intrinsic viscosity and degree of polymerization of cellulose. In addition, the impact of different dissolution times on the intrinsic viscosity of cellulose copper ethylenediamine when in contact with air was also discussed. This experiment is a basic characterization experiment of cellulose-related materials. By learning this experiment, students can understand the basic properties and dissolution mechanism of cellulose, as well as the determination of viscosity of polymer materials and the use of viscometer. They can also learn to build a simple model to explore the influence of different components.
\end{abstract}

Key Words: Cellulose; Lignin; Copper ethylenediamine; Intrinsic viscosity

高分子聚合物是单体分子通过聚合反应形成的相对分子量高达几千至几百万的化合物, 其聚合

收稿: 2020-10-28; 录用: 2020-11-23; 网络发表: 2020-12-23

*通讯作者, Emails: yueeryan@fudan.edu.cn (间玥儿); yitang@fuan.edu.cn (唐臨)

基金资助：国家自然科学基金(21805042，21802023); 上海市科委扬帆计划项目(18YF1401400，18YF1401900); 文化和旅游部文化行业标准化 研究项目(WH/Y08-2018); 上海市浦江人才计划(2020PJC003) 
度或分子量决定了高分子的加工和使用性能。测定高分子聚合度的方法包括凝胶色谱法、端基测定 法、光散射法、渗透压法和粘度法等。粘度法具有设备简单、操作便利、测定和数据处理周期短的 优势, 目前已被国内众多物理化学实验教材大量采用 ${ }^{[1-3]}$ 。粘度法可分为三类: 毛细管粘度法、落球 式粘度法、旋转粘度法等, 其中毛细管粘度法是使用最广泛的。但该方法只适用于稳定、可溶的纯 净高分子溶液, 所测分子量称为粘均相对分子质量。而在实际高聚物聚合度测量中, 也有很多高分 子由于不易溶解、溶解后不稳定或含有杂质等问题, 难于直接使用粘度法测定相对分子量。本文将 选择中国手工纸为测定对象, 针对其含有较多木质素杂质以及纤维素难溶解、易氧化降解等问题, 发展适合于化学类或纸质文物保护类本科生或者研究生实验课程的研讨型综合实验。

中国手工纸是通过传统工艺制备的以天然纤维素为主要成分的纸张, 其分子量测定是推断其使 役寿命和力学强度的基本参数, 对解决古籍断代、修复及再造意义重大。由于其主要成分纤维素纤 维含有大量氢键, 最常用的方法是采用铜乙二胺解氢键来制得适合粘度法测定的稀溶液 ${ }^{[4]}$ 。但在手 工纸的传统制备工艺中, 为了获得高纤维素得率和聚合度, 通常采取条件缓和的处理方法, 往往残 留一定量的木质素, 导致测定纤维素聚合度常用的铜乙二胺粘度法难以适用, 例如目前造纸领域的 现行国家标准要求所测纸浆木质素质量分数低于 $0.5 \%{ }^{[5]}$, 严重影响了对基于中国传统纸质文物的寿 命预测及保护材料的选择。此外在该法中, 由于铜乙二胺催化作用常常引起纤维素发生氧化反应, 导致纤维素分子量测定结果不准确, 因此应尽量避免溶液与氧气接触。

基于这些考虑, 本实验将构建铜乙二胺溶液的纤维素-木质素模型, 通过实验设计和数据分析, 探讨木质素含量对纤维素铜乙二胺法特性粘度及其分子量计算的影响, 并通过对不同手工纸样品的 实测, 具体了解实际复杂高分子聚合物体系分子量测定的过程。通过本实验, 学生将进一步提升对 高分子粘度法测定特性粘度和分子量的原理与方法的理解, 系统地掌握纤维素铜乙二胺法特性粘度 和聚合度的测定方法, 并学会探讨杂质或因素的影响。

\section{1 实验目的}

(1) 通过文献查阅, 了解纤维素的特点、研究现状和应用前景。

(2) 掌握高分子粘度法测定特性粘度和分子量的原理。

（3）掌握铜乙二胺法测定纤维素特性粘度和聚合度的原理及操作方法, 会系统讨论影响因素。

\section{2 实验原理}

\section{1 纤维素-铜乙二胺溶液配制}

纤维素具有多重氢键超分子结构, 且结晶态较复杂, 一般情况下不易溶于普通溶剂。铜乙二胺 配合物是一种纤维素的理想溶剂, 与纤维素 $\mathrm{C}_{2}$ 和 $\mathrm{C}_{3}$ 位的羟基发生强相互作用使纤维素溶解, 由此可 形成适合粘度测定的纤维素铜乙二胺溶液。纤维素溶于铜乙二胺的反应如下:

$$
\left(\mathrm{C}_{6} \mathrm{H}_{10} \mathrm{O}_{5}\right)_{2 n}+2 n\left[\mathrm{Cu}(\mathrm{en})_{2}\right](\mathrm{OH})_{2} \rightarrow\left[\left(\mathrm{C}_{6} \mathrm{H}_{8} \mathrm{O}_{5}\right)-\mathrm{Cu}(\mathrm{en})\right]_{2 n}+4 n \mathrm{H}_{2} \mathrm{O}+2 n(\mathrm{en})
$$

其中 $(e n)$ 代表乙二胺分子, 图1是纤维素溶解于铜乙二胺溶液后产物的分子结构示意图。

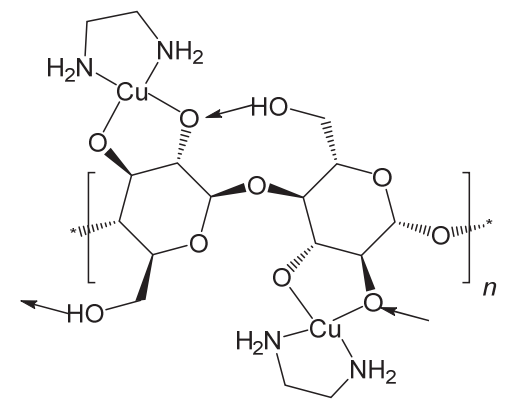

图1 纤维素溶解于铜乙二胺溶液后产物的分子结构示意图 


\section{2 纤维素-铜乙二胺溶液特性粘度测定及分子量}

纤维素-铜乙二胺溶液特性粘度测定的原理是先用毛细管粘度计测出上述预先配制的纤维素-铜 乙二胺溶液粘度 $(\eta)$ 相对于铜乙二胺溶剂粘度 $\left(\eta_{0}\right)$ 增加的分数, 即增比粘度 $\left(\eta_{\mathrm{sp}}\right)$, 然后采用马丁经验方 程(公式1), 即可得到其特性粘度 $[\eta]$ 。

$$
\eta_{\mathrm{sp}}=[\eta] \cdot \rho \cdot \mathrm{e}^{K[\eta] \rho}
$$

式中 $\rho$ 是纤维素在稀溶剂中的浓度 (以绝干计, 单位 $\mathrm{g} \cdot \mathrm{mL}^{-1}$ ); $K$ 为经验常数, 对于纤维素-铜乙二 胺系统, $K=0.13$ 。

继而再根据Mark-Houwink方程(公式 2 , 式中 $M$ 为高分子的分子量, $K$ 和 $\alpha$ 为与分子量无关的常数),

$$
[\eta]=K M^{\alpha}
$$

即可求得纤维素的分子量。通常以纤维素聚合度(DP)计，由公式2推得的经验公式3如下:

$$
\mathrm{DP}^{0.905}=0.75[\eta]
$$

\section{3 毛细管粘度计分类与选型根据}

毛细管粘度仪通常分为乌式粘度计、奥式粘度计和北欧标准粘度计三类, 如图2所示。其中奥氏 粘度计由于毛细管中的液体所受压力差与液面高度有关, 当标准液和待测液体积有差异时易造成误 差; 乌氏粘度计通过增加支管形成气承悬液柱, 既避免了测试液体积差异的影响, 并可以方便地在 粘度计储液球中调节溶液浓度, 所调配的溶液也可反复抽吸进行测试。但反复使用同一纤维素-铜乙 二胺溶液, 会增加该溶液与空气的接触, 导致纤维素分子量测定结果不准确, 因此乌氏粘度计并不 适合其粘度的测定。因此本实验选用北欧校准粘度计, 并舍弃每一次测试后溶液, 以避免纤维素氧 化降解的影响。

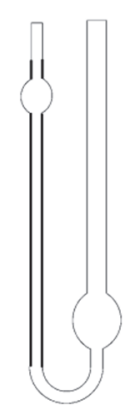

奥氏粘度计

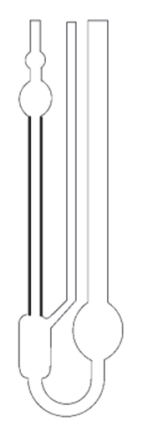

乌氏粘度计

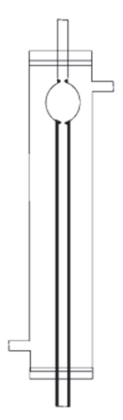

北欧标准粘度计

图2 常用毛细管粘度仪示意图

\section{3 试剂与仪器}

\section{1 试剂}

纤维素: 来源于浆粕, 由齐鲁工业大学省部共建生物基材料与绿色造纸国家重点实验室提供。 木质素：由上海阿拉丁生化科技股份有限公司生产。

铜乙二胺溶液: 按 GB/T 1548-2016《纸浆 铜乙二胺(CED)溶液中特性粘度值的测定》附录A《铜 乙二胺(CED)溶液的制备与分析》所述配制。

测试所用水为去离子水。

\section{2 仪器}

试样溶解瓶: 容积约为 $52 \mathrm{~mL}$ 的细口聚乙烯瓶, 当容器中装入 $50 \mathrm{~mL}$ 溶液时, 残留的空气能排出, 排出空气后盖好橡皮塞或带有密封垫的罗口盖。

毛细管粘度计: 带有水套的校准用毛细管粘度计和带有水套的测定用毛细管粘度计。具体要求 
参照GB/T 1548-2016《纸浆 铜乙二胺(CED)溶液中特性粘度值的测定》所用北欧标准粘度计。在测 定时, 要求特性粘度 $[\eta]$ 与纤维素浓度 $\rho$ 的乘积 $[\eta] \cdot \rho=3.0 \pm 0.5$, 并且粘度为 $11 \mathrm{mPa} \cdot \mathrm{s}$ 的溶液的流出时 间约为 $100 \mathrm{~s}$, 速度梯度 $G_{\max }$ 为 $(200 \pm 30) \mathrm{s}^{-1}$ 。 $G_{\max }$ 由公式 4 获得。

$$
G_{\max }=\frac{4 V}{\pi r^{3} t}
$$

式中, $V$ 为流出容积, $\mathrm{cm}^{3} ; r$ 为毛细管内半径, $\mathrm{cm} ; t$ 为流出时间, $\mathrm{S}$ 。

恒温水浴: 能控制在 $(25 \pm 1){ }^{\circ} \mathrm{C}$, 并装有自动循环泵。

天平: 精度 $0.001 \mathrm{~g}$ 。

振动器: 环境温度 $25^{\circ} \mathrm{C}$, 每分钟振动 250 次。

秒表: 精度为 0.01 秒。

\section{4 实验步骤}

\section{1 纤维素-木质素模型物的配制}

选取纤维素粉和木质素粉作为模型代表物, 称取纤维素 $1.40 \mathrm{~g}$, 分别称取相当于纤维素重量比为 $0 \% 、 1 \% 、 2 \% 、 4 \% 、 5 \% 、 10 \% 、 20 \% 、 30 \%$ 的木质素, 与纤维素一同加入溶解瓶中, 先加入 $25 \mathrm{~mL}$ 去 离子水, 使纤维素和木质素粉均匀分散, 然后加入 $25 \mathrm{~mL}$ 铜乙二胺溶液, 并加入2-3颗玻璃珠, 排除 空气后振荡溶解瓶, 使物料分散溶解。

\section{2 模型物特性粘度的测定}

测定 $(25 \pm 0.1)^{\circ} \mathrm{C}$ 下溶液自北欧标准粘度计上刻度线至下刻度线的流出时间, 用秒表计时, 测定 三次, 取其平均值计算表观相对粘度。根据表观相对粘度, 经查GB/T 1548-2016《纸浆 铜乙二胺 (CED) 溶液中特性粘度值的测定》附录 2 《不同粘度比 $\eta_{\mathrm{ratio}}$ 对应的 $[\eta] \times \rho$ 值》表格, 进一步得到表观特 性粘度 $[\eta]$ 。

\section{3 含有木质素的纸样特性粘度的测定}

(1) 将纸样撕成面积小于 $1 \mathrm{~cm}^{2}$ 的碎片。根据 GB/T 1462-2008《纸、纸板和纸浆 分析试样水分的 测定》测定纸样水分。根据GB/T 747-2003《纸浆 酸不溶木质素的测定》和GB/T 10337-2008《造纸 原料和纸浆 酸溶木质素的测定》测定纸样中木质素总含量。

(2) 取一定量试样(精确至 $0.005 \mathrm{~g}$ )于溶解瓶中, 先加入 $25 \mathrm{~mL}$ 去离子水, 剧烈震荡使试样均匀分 散, 并浸泡至少 $2 \mathrm{~h}$, 使纤维充分润胀, 然后加入 $25 \mathrm{~mL}$ 铜乙二胺溶液, 并加入2-3颗玻璃珠, 排除空 气后振荡溶解瓶, 使试样溶解。

(3) 调整溶液温度至 $25^{\circ} \mathrm{C}$, 倒出溶液于测定用毛细管粘度计中, 启动自动循环保温的恒温器, 恒温 5 min。

(4) 测定 $(25 \pm 0.1)^{\circ} \mathrm{C}$ 下溶液自毛细管粘度计上刻度线至下刻度线的流出时间, 用秒表计时, 测 定三次, 取其平均值计算表观相对粘度。根据表观相对粘度, 经查GB/T 1548-2016《纸浆 铜乙二胺 (CED) 溶液中特性粘度值的测定》附录 2 《不同粘度比 $\eta_{\text {ratio }}$ 对应的 $[\eta] \times \rho$ 值》表格, 进一步得到表观特 性粘度 $[\eta]$ 。

\section{5 实验结果举例}

\section{1 木质素含量对纤维素铜乙二胺特性粘度的影响}

图3是含不同木质素的纤维素铜乙二胺溶液的特性粘度测定结果。由图3可知, 提高木质素含量, 会导致模型物的表观特性粘度值下降, 并与木质素含量呈线性关系(如图3中圆点所示)。在扣除木质 素含量后, 可以获得仅以纤维素实际浓度计的特性粘度, 我们将该粘度称为校正特性粘度(如图3中 菱形所示), 可见, 该粘度值不随木质素含量的增加而变化, 且与木质素为 $0 \%$ 条件下的纤维素的特性 粘度值相当, 说明通过纤维素的实际浓度来计算其铜乙二胺溶液的特性粘度可以排除木质素含量的 
影响, 获得准确的特性粘度值。此外, 尽管该模型设计了木质素 $30 \%$ 含量的实验, 但实际操作中, 当 木质素含量超过 $20 \%$ 后, 有堵塞粘度计毛细管的情况, 此时所测结果已不再准确。

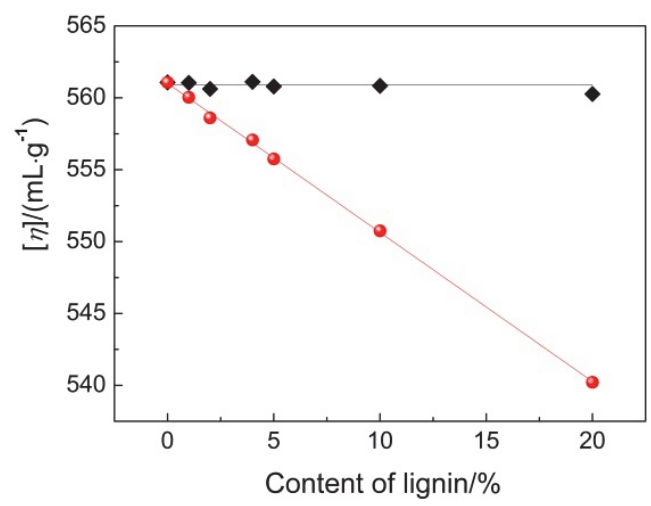

图3 木质素含量对纤维素铜乙二胺法所得特性粘度的影响[6]

- 表观特性粘度; ・校正特性粘度

\section{2 纸张样品特性粘度讨论}

本实验选择了 3 种木质素含量不同的纸样, 并基于上述方法测定了纸样中纤维素的校正特性粘 度, 进一步根据方程(2)获得了纸样纤维素的聚合, 所得结果列于表1中。这一方法不仅降低了木质素 的影响, 同时获得了更加准确的特性粘度值和聚合度, 能够降低由木质素影响而导致的纤维素聚合 度偏差，进而为纸张的寿命预测提供更好的依据 ${ }^{[7,8]}$ 。

表13种纸样铜乙二胺法特性粘度和聚合度的测试结果 ${ }^{[6]}$

\begin{tabular}{ccccc}
\hline 纸张种类 & 木质素含量 $/ \%$ & 表观特性粘度 $/\left(\mathrm{mL} \cdot \mathrm{g}^{-1}\right)$ & 校正特性粘度 $/\left(\mathrm{mL} \cdot \mathrm{g}^{-1}\right)$ & 聚合度 \\
\hline 纸样 & 1.24 & 1403 & 1412 & 2200 \\
纸样2 & 5.05 & 886 & 940 & 1400 \\
纸样3 & 0.49 & 308 & 327 & 440 \\
\hline
\end{tabular}

\section{3 纤维素特性粘度氧化降解的讨论}

图4显示了空气接触下不同溶解时间对纤维素铜乙二胺特性粘度的影响。如图4所示, 纤维素铜 乙二胺溶液接触空气后, 特性粘度值随溶解时间的延长而逐渐下降。为了与北欧标准粘度计作对比, 本实验还选用了乌氏粘度计进行测试, 也出现了纤维素特性粘度下降的现象, 这是纤维素溶液经多 次回用出现降解的结果。所以, 为了得到可靠而稳定的结果, 应尽量避免纤维素铜乙二胺溶液与空 气的接触, 而且, 待测溶液在使用一次后应弃置, 不再回用。

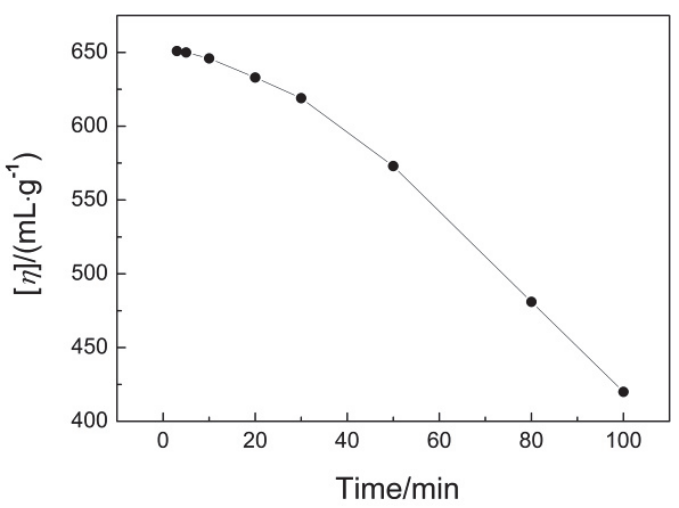

图4 空气接触下不同溶解时间对纤维素铜乙二胺特性粘度的影响 


\section{6 实验注意事项}

本实验涉及纤维素的相关知识, 包括纤维素的溶解和特性粘度测定, 在实验操作中, 需要注意 以下几点:

(1) 在配制铜乙二胺溶液时注意温度和化学品用量的控制, 如不方便配制, 可直接购买铜乙二 胺溶液, 之后根据国家相关标准进行标定。

(2) 试样和溶剂装入溶解瓶后, 应尽量排除空气, 避免氧气引起试样降解。

(3) 在测试模型物特性粘度时, 注意木质素含量超过 $20 \%$ 后, 有堵塞粘度计毛细管的情况, 此时 所测结果已不再准确。而当纸张的木质素含量超过 $10 \%$ 后, 已不能再使用 CED法测定其特性粘度和 聚合度。

(4) 对于纸张纤维, 尤其是纤维较长的情况, 在铜乙二胺溶液中溶解需要一个过程。可根据木质 素含量的差异采取不同的溶解方式, 木质素含量高的试样, 溶解时间延长。溶解方式可选择在溶解 瓶中放置转子、玻璃珠等帮助搅拌, 也可在振动器上进行。

(5) 与一般高分子溶液测试不同, 经过测试的纤维素铜乙二胺溶液已经被空气氧化, 该溶液不 可回用。

\section{7 思考题}

(1) 纤维素与常见高分子材料有何差别? 为什么不溶于一般有机溶剂?

(2) 模型物的特性粘度与木质素含量有什么关系?

(3) 纤维素的铜乙二胺溶液如果不排空气会对结果有什么影响?

(4) 评价利用粘度法测定高分子分子量的优缺点, 讨论影响测试结果的因素。

\section{8 结语}

本文介绍了铜乙二胺法测定纤维素特性粘度的改进实验, 并探讨了木质素含量对纤维素铜乙二 胺法特性粘度的影响。本实验不仅涉及到纤维素的溶解、特性粘度的测试和氧化降解的影响, 还讨 论了木质素含量对纤维素铜乙二胺溶液特性粘度的影响。通过构建的纤维素-木质素模型物, 扣除木 质素含量, 获得仅以纤维素实际浓度计的校正特性粘度, 进而获得更准确的纤维素聚合度值, 对同 时含有木质素和纤维素的天然纤维的测试有着重要意义。本实验可让学生深入理解高分子的粘度测 定原理和方法, 在学习和实践中构建模型思维, 延伸到实际样品分析, 有助于充分发挥实践与理论 相结合的优势, 提高综合能力。

\section{参 考 文 献}

[1] 复旦大学等, 编, 庄继华等, 修订. 物理化学实验. 第3版. 北京: 高等教育出版社, 2004.

[2] 郭玲香, 宁春花, 主编. 高分子化学与物理实验. 南京: 南京大学出版社, 2014.

[3] 崔玉红, 赵占芬, 梁山, 陈京, 赵妍, 主编. 基础物理化学实验. 天津: 天津大学出版社, 2018.

[4] 薛玉, 董正祥, 张晓显, 张东光. 造纸化学品, 2015, 27 (4), 29.

[5] 中国国家标准化管理委员会. 纸浆 铜乙二胺(CED)溶液中特性粘度值的测定: GB/T1548-2016. 北京: 中国标准出版社, 2016.

[6] 刘鹏, 章若红, 江艳, 刘峻, 孙梦捷, 间玥儿, 唐卧. 文物保护与考古科学, 2019,31 (6), 15.

[7] Zou, X.; Uesaka, T.; Gurnagul, N. Cellulose 1996, 3, 243.

[8] Coppola, F.; Modellii, A. Cellulose 2020, 27, 8977. 